\title{
Antidepressant Prescription Medication Use Patterns among Insured, Low Income Pregnant Women
}

\author{
Jun $\mathrm{Wu}^{1, *}$, Mary Lynn Davis-Ajami ${ }^{2}$, Sharon Keiser ${ }^{3}$, Lynda Sykes ${ }^{4}$ \\ ${ }^{1}$ South Carolina College of Pharmacy, University of South Carolina, Greenville, USA \\ ${ }^{2}$ Organizational Systems and Adult Health, School of Nursing, University of Maryland, Baltimore, Baltimore, USA \\ ${ }^{3}$ Sharon Keiser, Department of Obstetrics and Gynecology, Greenville Health System, Greenville, USA \\ ${ }^{4}$ Lynda Sykes, Department of Pharmacy, Greenville Health System, Greenville, USA \\ *Corresponding author: wujun@sccp.sc.edu
}

Received December 29, 2012; Revised April 23, 2013; Accepted April 28, 2013

\begin{abstract}
Despite of the controversy of antidepressant use during the gestational period, the reported antidepressant use at any time during pregnancy has increased. Our study assessed antidepressant prescription medication use patterns during pregnancy among insured, low-income women in the United States. This retrospective study used South Carolina Medicaid claims data (2004-2009). Included were pregnant women $(\geq 18$ years) with single or multiparous live birth(s) with a diagnosis for major depression or reported pharmacy claims for antidepressant prescriptions 1 year before becoming pregnant or while pregnant. Antidepressant prevalence prior to and during pregnancy, type of antidepressant, and trimester when the antidepressant use started, characterized antidepressant prescription use patterns. Among 24,077 pregnant women with live births, the prevalence of antidepressant use before becoming pregnant and during pregnancy was 5.5\% and 6\% respectively. A total of 3,237 pregnant women met inclusion criteria. Of the 1,456 women prescribed antidepressants during pregnancy, $16 \%$ showed a history of major depression predating their pregnancy and $67 \%$ initiated antidepressant medications after becoming pregnant. Nearly $80 \%$ took selective serotonin reuptake inhibitors (SSRIs) during pregnancy, and 32\% initiated antidepressants during the first trimester. Age, race, and preexisting depression were associated with antidepressant use. In pregnant, insured low-income women, the majority prescribed antidepressants were new users. Pharmacologic treatment for pregnant women with depressive symptoms, particularly in newly diagnosed major depression remains challenging.
\end{abstract}

Keywords: antidepressant, insured, low-income, pregnancy, depression

\section{Introduction}

Depression affects approximately one in ten U.S. adults and is associated with negative health outcomes in several chronic diseases, such as arthritis, asthma, cardiovascular disease, cancer, diabetes, and obesity [1,2,3,4,5]. According to a report by the American Congress of Obstetricians and Gynecologists, the percentage of women experiencing depressive disorders during pregnancy ranges from $14 \%$ to $23 \%$ [6]. This suggests many women may take an antidepressant at some time during pregnancy [7]. However, pregnant women with depression face complicated treatment decisions due to either the risk associated with not treating depression or the risks associated with antidepressant use. Studies report both depressive symptoms and antidepressant exposure during pregnancy as being associated with fetal growth changes and shorter gestations $[7,8,9,10]$. In addition, women who discontinue antidepressant during pregnancy are more likely to experience relapse of depression and withdrawal symptoms, which might affect both maternal and newborn outcomes $[11,12,13]$. Understanding patterns of antidepressant use in pregnant women may assist clinicians in developing antidepressant treatment plan to optimize outcomes.
Low socio-economic status is associated with depression. Studies show individuals who have less than a high school education, are unable to work, are unemployed, or are without health insurance are more likely to have more severe depressive symptoms [2,14,15]. Hayes et al report that antidepressant use during pregnancy for women enrolled in Medicaid as being associated with preterm birth and infant convulsions [16]. However, there is limited information from most recent year data regarding antidepressant prescription medication use to treat major depression during pregnancy in underprivileged populations. Thus, understanding antidepressant medication use patterns in low-income pregnant women may assist policymakers and health care professionals in identifying obstacles to depressive disorders management during pregnancy and identifying factors important to devising individualized care and improving depressive disorder outcomes in women during pregnancy.

To address medication use as a depression treatment option in low-income pregnant women, we utilized South Carolina Medicaid claims data to identify a patient population that would most closely represent our target population. We hypothesize that patient characteristics including demographics and previous clinical history and 
medical utilization before pregnancy might affect the receipt of maternal antidepressant treatment. Our objectives were (1) to describe antidepressant prescription medication use patterns in low-income, insured women during pregnancy; (2) to identify patient characteristics that predict the likelihood of antidepressant use during pregnancy.

\section{Methods}

This was a retrospective cohort study covering the years 2004-2009 among South Carolina Medicaid female enrollees ages 18 years and older, with single or multiparous live births during the study period and who showed a primary diagnosis for major depression or used antidepressants during the entire time in the one year prior to becoming pregnant or at any time during pregnancy. The date of pregnancy was calculated as 280 days prior to a maternal delivery. The pregnant women included in our study were required to show continuous Medicaid enrollment at least 1 year before and 1 year after pregnancy. Women with diagnosis for schizophrenia or bipolar disorders, as well as those with any antipsychotic medication claim during the eligibility period were excluded from the study. All medical conditions were identified from medical claims using the International Classification of Diseases, 9th revision, Clinical Modification (ICD-9-CM) codes. Antidepressant prescription medications were identified from pharmacy claims by national drug codes. If a woman had more than one maternal delivery during the study period, the first delivery meeting eligibility criteria was included for analysis.

Individuals were followed from the calculated date of conception until delivery. Women with a primary diagnosis of major depression at any time during the 365 days before the calculated date of conception were defined as patients with a history of depression pre-dating pregnancy. Women with at least one antidepressant claim in one year before pregnancy were defined as patients with history of antidepressant use before pregnancy. Women with a primary diagnosis of major depression during pregnancy were defined as patients with depression during pregnancy. Women with at least one antidepressant claim from the calculated date of conception until the date of delivery were defined as patients using antidepressants during pregnancy. We divided the cohort into four groups: (1) women with a major depression diagnosis and antidepressant prescription medication use during pregnancy; (2) women with a major depression diagnosis and no antidepressant prescription medication use during pregnancy; (3) women without a diagnosis for major depression and who used antidepressant prescription medications during pregnancy; (4) women without major depression diagnosis and who did not use antidepressant prescription medications during pregnancy.

This study assessed antidepressant prescription medication use patterns during pregnancy among the aforementioned four groups, by focusing on: (1) antidepressant prescription medication use by drug class; (2) total number of antidepressant prescription medications used; and (3) trimester the pregnant woman initiated antidepressant prescription medication use. The major outcome of interest was the likelihood of antidepressant use during pregnancy. Potential predictors assessed included age, race, depression and antidepressant use history before becoming pregnant, comorbidity, and medical utilization at any time during the 365 days before the calculated date of conception. The Charlson comorbidity index was used to create a weighted score (ranging from 1-6) to assess comorbidity severity. ICD-9$\mathrm{CM}$ codes identified comorbid conditions from Medicaid claims data.

Descriptive and basic statistics (one way ANOVA tests for continuous variables and Chi-square $\left(\chi^{2}\right)$ tests for categorical variables) were used to describe and compare characteristics and antidepressant use patterns from the calculated date of conception until the date of delivery for the study population. A logistic regression model was applied to identify significant predictors associated with antidepressant use during pregnancy. The level of statistical significance was set at $\alpha=0.05$ for all analyses. All data analyses were performed using SAS 9.2 (SAS Institute, Cary, NC). The study protocol was approved by the Institutional Review Board at University of South Carolina.

\section{Results}

The South Carolina Medicaid claims data reported a total of 24,077 live births in the period between January 1, 2004 and December 31, 2009 in women with continuous enrollment in Medicaid one year before and one year after pregnancy. Of those, 1344 women (5.5\%) used antidepressants before becoming pregnant and 1456 women $(6.0 \%)$ had exposure to antidepressants during pregnancy. Of the 3,237 pregnant women who met our inclusion criteria (Figure 1), 13.5\% ( $\mathrm{n}=438)$ were diagnosed with major depression and used antidepressants during pregnancy (group 1), 27.4\% ( $\mathrm{n}=887)$ were diagnosed with major depression and did not use antidepressants during pregnancy (group 2), 31.4\% ( $\mathrm{n}=$ 1018) were not diagnosed with major depression but used antidepressants during pregnancy (group 3), and the rest $27.6 \%(\mathrm{n}=894)$ were not diagnosed with major depression and did not use antidepressants during pregnancy either (group 4). Baseline characteristics of the study population are shown in Table 1 . Of 1456 women who used antidepressants during pregnancy (groups 1 and $3), 16 \%(n=236)$ had history of a diagnosis for major depression and 33\% $(n=484)$ used antidepressants before becoming pregnant. Of 1718 women who did not use antidepressants during pregnancy (groups 2 and 4), 34\% $(\mathrm{n}=580)$ had history of a diagnosis for major depression and 50\% $(n=860)$ used antidepressants before becoming pregnant. Those included were predominately Caucasian ( $72 \%$ ), and averaged 25 years of age at the date of live birth delivery. Medical utilization during the one year before pregnancy was significantly different among all four groups.

Antidepressant use patterns during pregnancy results are shown in Table 2. Of the 1456 women who used antidepressants during pregnancy, 30\% $(n=438)$ had a diagnosis of major depression, nearly $75 \%$ used SSRI, and $14 \%$ used two or more antidepressants. Thirty two percent, $43 \%$ and $25 \%$ of women initiated antidepressant 
pharmacologic intervention at the first, second, and third trimesters during pregnancy respectively. Women with a diagnosis of major depression during pregnancy appear to be more likely to initiate antidepressant prescription medications in the first trimester.

Results of the logistic regression model are shown in Table 3. After adjusting for all other confounders, every one year increase in age was associated with a 5\% increase in the odds of antidepressant use during pregnancy. Non-Caucasian women were 59\% less likely to use antidepressants during pregnancy than Caucasian women. Women with a history of a major depression diagnosis before becoming pregnant showed 35\% lower odds of antidepressant use during pregnancy. The likelihood of antidepressant use during pregnancy was reduced by $20 \%$ in women with at least one emergency department (ED) visit before becoming pregnant.

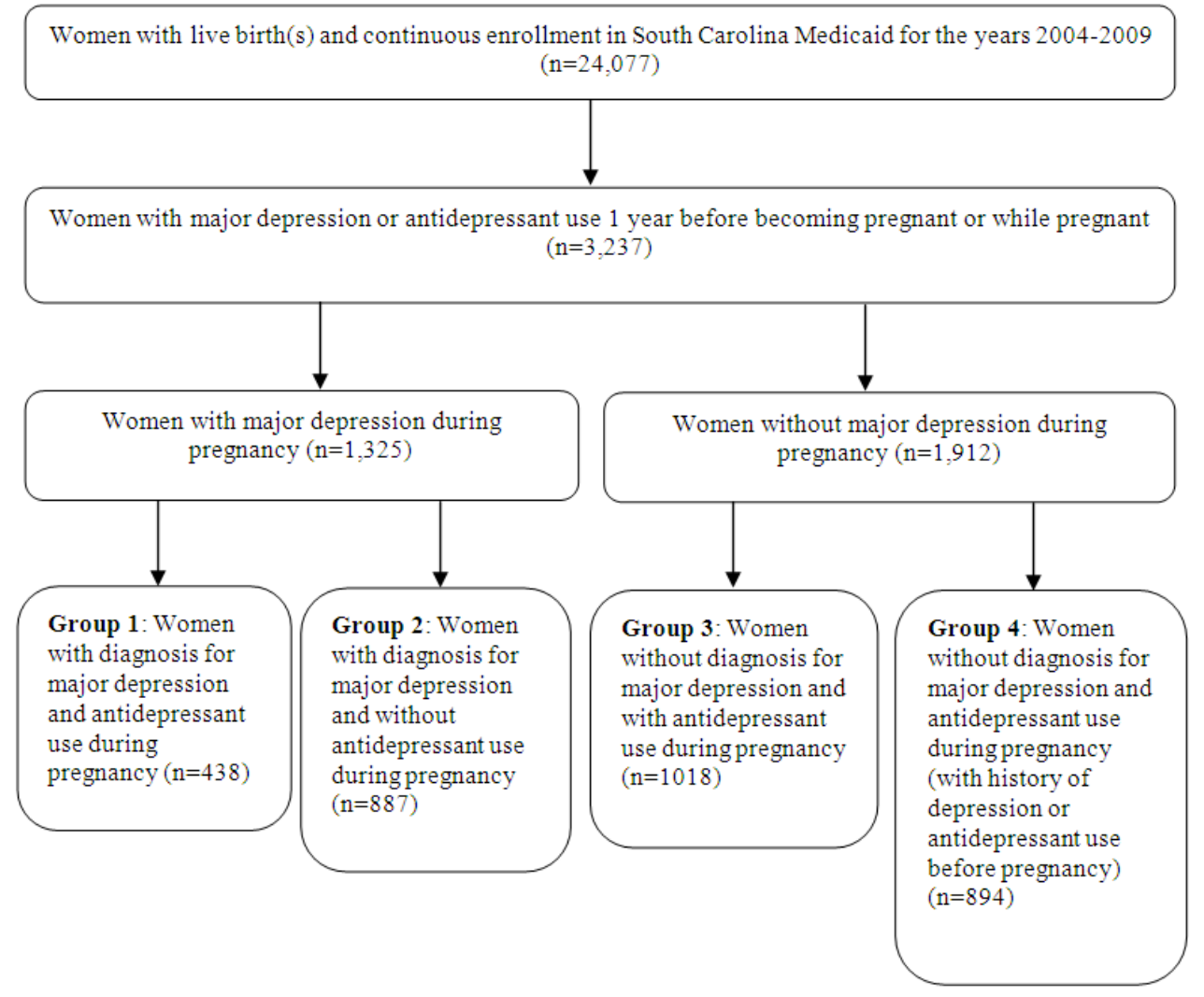

Figure 1. Patient selection

Table 1. Characteristics of study population

\begin{tabular}{|c|c|c|c|c|c|c|}
\hline Variable & All $(n=3237)$ & $\begin{array}{l}\text { Group } 1 * \\
(\mathrm{n}=438)\end{array}$ & $\begin{array}{l}\text { Group } 2 * \\
(\mathrm{n}=887)\end{array}$ & $\begin{array}{l}\text { Group } 3 * \\
(\mathrm{n}=1018)\end{array}$ & $\begin{array}{c}\text { Group } 4 * \\
(\mathrm{n}=894)\end{array}$ & P-value \\
\hline Age, years, Mean (SD) & $24.7(5.8)$ & $25.5(5.6)$ & $24.3(5.5)$ & $25.3(6.0)$ & $24.0(5.5)$ & $<0.001$ \\
\hline$\leq 25, \mathrm{n}(\%)$ & $2060(63.6)$ & $251(57.3)$ & $601(67.8)$ & $609(59.8)$ & $599(67.0)$ & $<0.001$ \\
\hline$>25, \mathrm{n}(\%)$ & $1177(36.4)$ & $187(42.7)$ & $286(32.2)$ & $409(40.2)$ & $295(33.0)$ & \\
\hline Race, $\mathrm{n}(\%)$ & & & & & & $<0.001$ \\
\hline Caucasian & $2337(72.2)$ & $350(79.9)$ & $612(69.0)$ & $808(79.4)$ & $567(63.4)$ & \\
\hline African American & 840 (25.9) & $72(16.4)$ & $264(29.8)$ & $190(18.7)$ & $314(35.1)$ & \\
\hline Other & $60(1.9)$ & $16(3.7)$ & $11(1.2)$ & $20(1.9)$ & $13(1.5)$ & \\
\hline $\begin{array}{l}\text { Charlson comorbidity score, mean } \\
\text { (SD) }\end{array}$ & $0.12(0.75)$ & $0.10(0.69)$ & $0.07(0.56)$ & $0.10(0.70)$ & $0.19(0.96)$ & 0.005 \\
\hline $0-2, \mathrm{n}(\%)$ & $3188(98.5)$ & $434(99.1)$ & $879(99.1)$ & $1003(98.5)$ & $872(97.5)$ & 0.178 \\
\hline $3-4, \mathrm{n}(\%)$ & $28(0.9)$ & $2(0.5)$ & $5(0.6)$ & $9(0.9)$ & $12(1.4)$ & \\
\hline$\geq 5, \mathrm{n}(\%)$ & $21(0.7)$ & $2(0.4)$ & $3(0.3)$ & $6(0.6)$ & $10(1.1)$ & \\
\hline \multicolumn{7}{|l|}{$\begin{array}{l}\text { Medical utilization in } 1 \text { year before } \\
\text { pregnancy }\end{array}$} \\
\hline Office visit , mean (SD) & $4.6(6.0)$ & $4.5(6.8)$ & $3.8(5.6)$ & $3.6(5.9)$ & $6.1(5.6)$ & $<0.001$ \\
\hline Outpatient visits, mean (SD) & $1.4(2.8)$ & $1.4(3.0)$ & $1.0(2.4)$ & $1.3(3.1)$ & $1.7(2.6)$ & $<0.001$ \\
\hline Emergency room, n(\%) & $1338(41.3)$ & $183(41.8)$ & $302(34.1)$ & $330(32.4)$ & $523(58.5)$ & $<0.001$ \\
\hline Hospitalization, $\mathrm{n}(\%)$ & 613 (18.9) & 87 (19.9) & $152(17.1)$ & $163(16.0)$ & $211(23.6)$ & $<0.001$ \\
\hline \multicolumn{7}{|l|}{$\begin{array}{l}\text { History of depression and } \\
\text { antidepressant use in } 1 \text { year before } \\
\text { pregnancy }\end{array}$} \\
\hline Diagnosis of depression, $\mathrm{n}(\%)$ & $816(25.2)$ & $121(27.6)$ & $148(16.7)$ & $115(11.3)$ & $432(48.3)$ & $<0.001$ \\
\hline Antidepressant use, $\mathrm{n}(\%)$ & $1344(41.5)$ & $192(43.8)$ & $148(16.7)$ & $292(28.7)$ & $712(79.6)$ & $<0.001$ \\
\hline
\end{tabular}

*Group 1: Women with depression diagnosis and antidepressant use during pregnancy; Group 2: Women with depression diagnosis and without antidepressant use during pregnancy; Group 3: Women without depression diagnosis and with antidepressant use; Group 4: Women without depression diagnosis and antidepressant use during pregnancy (with history of depression or antidepressant use before pregnancy)

SD: Standard deviation 
Table 2. Antidepressant use patterns in women during pregnancy

\begin{tabular}{|c|c|c|c|c|}
\hline Variable & All $(n=1456)$ & $\begin{array}{l}\text { With depression diagnosis } \\
\qquad(\mathrm{n}=438)\end{array}$ & $\begin{array}{l}\text { Without depression diagnosis } \\
\qquad(\mathrm{n}=1018)\end{array}$ & P-value \\
\hline \multicolumn{5}{|l|}{ Type of antidepressant use } \\
\hline SSRI & $1071(73.6)$ & $340(77.6)$ & $731(71.8)$ & 0.021 \\
\hline SNRI & $105(7.2)$ & $51(11.7)$ & $54(5.3)$ & $<0.001$ \\
\hline TCA & $103(7.0)$ & $34(7.8)$ & $69(6.8)$ & 0.502 \\
\hline Miscellaneous & $335(23.0)$ & $95(21.7)$ & $240(23.6)$ & 0.433 \\
\hline No. of antidepressant use & & & & $<0.001$ \\
\hline 1 & $1260(86.5)$ & $351(80.2)$ & 909 (89.3) & \\
\hline 2 & $173(11.9)$ & $75(17.1)$ & $98(9.6)$ & \\
\hline$>2$ & $23(1.6)$ & $12(2.7)$ & $11(1.1)$ & \\
\hline Trimester initiating therapy & & & & $<0.001$ \\
\hline 1st trimester & $467(32.1)$ & $182(41.6)$ & $285(28.0)$ & \\
\hline 2nd trimester & $627(43.1)$ & $164(37.4)$ & $463(45.5)$ & \\
\hline 3rd trimester & $362(24.9)$ & $92(21.0)$ & $270(26.5)$ & \\
\hline
\end{tabular}

Table 3. Association between potential predictors and antidepressant use during pregnancy $(\mathbf{n}=\mathbf{3 2 3 7})$

\begin{tabular}{|c|c|}
\hline Independent variable & $\begin{array}{c}\text { Dependent variable: likelihood of antidepressant use during pregnancy } \\
\text { Odds ratio (95\% confidence interval) }\end{array}$ \\
\hline Age (years) & $1.05(1.03,1.06)^{* * *}$ \\
\hline \multicolumn{2}{|l|}{ Race } \\
\hline Caucasian & 1.00 \\
\hline Non-Caucasian ${ }^{1}$ & $0.41(0.33,0.50)^{* * *}$ \\
\hline \multicolumn{2}{|c|}{ Previous diagnosis of depression in 1 year before pregnancy } \\
\hline No & 1.00 \\
\hline Yes & $0.65(0.52,0.81)^{* * *}$ \\
\hline \multicolumn{2}{|c|}{ Previous antidepressant use in 1 year before pregnancy } \\
\hline No & 1.00 \\
\hline Yes & $1.00(0.83,1.20)$ \\
\hline Charlson Comorbidity score & $1.03(0.92,1.15)$ \\
\hline \multicolumn{2}{|c|}{ Medical utilization in 1 year before pregnancy } \\
\hline \multicolumn{2}{|l|}{ Hospitalization } \\
\hline No & 1.00 \\
\hline Yes & $0.91(0.70,1.18)$ \\
\hline \multicolumn{2}{|l|}{ Emergency room } \\
\hline No & 1.00 \\
\hline Yes & $0.80(0.66,0.98)^{* *}$ \\
\hline Total number of office visits & $0.99(0.97,1.01)$ \\
\hline Total number of outpatient visit & $1.05(1.02,1.09)^{* *}$ \\
\hline
\end{tabular}

1. Non-Caucasian included African American and other races.

$*: \mathrm{p}<0.05, * *: \mathrm{p}<0.01, * * *: \mathrm{p}<0.001$

\section{Discussion}

Despite potential increased risk for adverse effects with antidepressant exposure for both mothers and unborn babies, our findings indicate that $6 \%$ of low-income, insured women giving birth used antidepressants during pregnancy. In addition, our study identified predictors associated with antidepressant use in low income, insured pregnant women. Learning more about the magnitude and predictors of gestational antidepressant use in Medicaid enrolled pregnant women may impact depressive symptom management in pregnant women, as well as help in the design and evaluation of appropriate anti-depression pharmacotherapy interventions and treatment modalities in this disadvantaged population.
We found similar prevalence of antidepressant use by women during pregnancy to previous studies using various administrative claims data, where $4-10 \%$ women reported antidepressant use during pregnancy. $[17,18,19,20]$ For example, Cooper et al reported $8.7 \%$ of women giving birth had exposure to any antidepressant in a Medicaid population during 1999-2003.(20)] Andrade and colleagues in a study using HMO claims data for the years 2001-2005 found 6.6\% of women were dispensed an antidepressant [19]. Although previous studies reported potentially increased risk for adverse effects on maternal and fetal outcomes related to exposure to SSRIs during pregnancy, [21-26] SSRIs were the most frequently prescribed class of antidepressant in our study. Previous studies also reported a decline in antidepressant use after women became pregnant, especially during the first trimester. $[16,27,28]$ Our study showed less than $35 \%$ of 
women taking antidepressants during pregnancy had history of antidepressant use before pregnancy. However, this does not mean overall antidepressant use during the entire gestational period was reduced because our findings showed two thirds of our sample newly initiated pharmacologic treatment after becoming pregnant. Moreover, nearly $70 \%$ of women receiving antidepressant drug therapy during pregnancy initiated pharmacologic treatment during the second or third trimester. Our study did not observe a significant decrease in overall antidepressant use during pregnancy. This suggests antidepressant drug therapy plays an important role in controlling depressive symptoms in pregnant women with newly diagnosed depression.

Notably, for the 1456 women receiving antidepressants during pregnancy (groups 1 and 3), nearly $70 \%$ were not diagnosed with major depression, suggesting that gestational depression was under diagnosed or antidepressants were prescribed to treat comorbid depressive symptoms or other conditions. Alternately, for the 1325 women with a major depression diagnosis during their pregnancy (groups 1 and 2), two thirds did not receive antidepressant drug therapy. Behavioral treatment, such as support groups or private psychotherapy, might be used with pregnant women with mild to moderate depression to manage their symptoms. To some extent, this result may reflect an awareness of the risks associated with taking antidepressants during pregnancy such as poor maternal and fetal health outcomes. Arguably, since the Medicaid population represents people with low socioeconomic status, this result could also be interpreted as insufficient treatment received by low-income pregnant women with depression due to some barriers to accessing health care services. Thus, further studies are needed to evaluate the association between severity of depression and treatment received by low-income pregnant women.

Our study identified age, race, history of depression during the 1 year before pregnancy, and medical utilization before pregnancy (ED and outpatient visits) as significant predictors associated with antidepressant use during pregnancy. Our analysis showed older aged pregnant women more likely to use an antidepressant. Additionally, we observed racial differences between Caucasian and non-Caucasian races in antidepressant use during pregnancy. This observed racial disparity is concerning because African Americans, Hispanics, and non-Hispanic persons of other races are more likely to meet the criteria for major depression. [2,29,30] These racial disparities may also have reflected differences in depression treatment preference and perceived barriers to treatment for depression during pregnancy between Caucasian and non-Caucasian pregnant women. [31,31,33] Women with a history of depression before pregnancy were less likely to receive an antidepressant. Some women with mild-too-moderate depression during the post-partum period may have switched to psychosocial approaches such as individual and group psychotherapy. Or, depressive symptoms may not have resurfaced during pregnancy in women previously receiving antidepressants. This suggests a possible correlation between adherence to antidepressant treatments to control depressive symptoms before pregnancy and reduced relapse of depression or odds of antidepressant use during pregnancy. [11,34]
In terms of medical utilization, patients with at least one ED visit before pregnancy were less likely to take antidepressants during pregnancy, suggesting perceptions about serious or acute medical events may have influenced health beliefs or motivated health behaviors. By contrast, having a greater number of outpatient visits increased the odds of using antidepressant during pregnancy, suggesting that access to outpatient care or possibly other socioeconomic factors influenced these women to initiate or continue antidepressant use.

These results based on Medicaid claims data should be interpreted in light of some limitations. First, the study cohort consisted of pregnant women enrolled in South Carolina Medicaid. Although this population was representative of low-income pregnant women in the United States, especially those with major depressive disorders or those receiving antidepressant pharmacotherapy, other studies may have observed different results in a more affluent cohort using a national level database. Secondly, antidepressant use estimated by administrative claims data may reflect prescribing patterns instead of actual medication use in pregnant women. We could not obtain accurate information about compliance with prescribed regimens. Moreover, using an average of 280 days from the date of delivery to estimate the date of conception may have misclassified antidepressant use and depression diagnosis before or after pregnancy. Finally, these claims data could not measure the severity of depression or the intensity of non-pharmacological intervention received by pregnant women. In addition, we could not assess the role of behavioral or psychological approaches to treat depressive symptoms in women during pregnancy and the association between gestational antidepressant use and severity of depression.

Despite limitations, our findings have implications for health care professionals and other policymakers responsible for the appropriate use of antidepressants in pregnant women. Although debate continues over the safety and long-term effects of antidepressant medication use during pregnancy, maintenance pharmacotherapy remains recommended for pregnant woman with severe or highly recurrent depression. [6,7,9] However, our study found a majority of low-income women initiating antidepressant treatments during pregnancy were new antidepressant users or had no primary diagnosis for major depression during pregnancy. Thus, Diagnosis and appropriate management of depression during pregnancy is an important part of prenatal care and should be addressed by clinicians, particularly reproductive health practitioners. Assessing risk and benefits of gestational antidepressant use, monitoring maternal and birth outcomes, and understanding antidepressant use behavior in low-income pregnant women are necessary to optimize treatment and deserve further study.

\section{Acknowledgement}

This study was funded by Institute for Advancement of Health Care, Greenville Health System and University of South Carolina.

\section{Statement of Interest}

There are no conflicts of interest to report. 


\section{List of Abbreviations}

ED: emergency department

SNRI: Serotonin-norepinephrine reuptake inhibitor

SSRI: selective serotonin reuptake inhibitor

TCA: Tricyclic antidepressant

\section{References}

[1] Mok, C., Lok, E. and Cheung, E, "Concurrent psychiatric disorders are associated with significantly poorer quality of life in patients with rheumatoid arthritis," Scand J Rheumatol, 41(4), 253-259, 2012.

[2] Centers for Disease Control and Prevention, "Current depression among adults --- united states, 2006 and 2008," Morbidity and Mortality Weekly Report (MMWR), 59(38), 1229-1235, 2010.

[3] Campayo, A., de Jonge P, Roy J.F., Saz, P., de la Camara. C., Quintanilla, M.A., Marcos,G., Santabarbara, J. and Lobo, A, "Depressive disorder and incident diabetes mellitus: The effect of characteristics of depression," Am J Psychiatry, 167(5), 580-588, 2010.

[4] Lichtman, J.H., Bigger, J.T,Jr, Blumenthal, J.A., at el. "Depression and coronary heart disease: Recommendations for screening, referral, and treatment: A science advisory from the american heart association prevention committee of the council on cardiovascular nursing, council on clinical cardiology, council on epidemiology and prevention, and interdisciplinary council on quality of care and outcomes research: Endorsed by the american psychiatric association," Circulation, 118(17),1768-1775, 2008.

[5] Campayo, A., Gomez-Biel, C.H. and Lobo, A, "Diabetes and depression," Curr Psychiatry Rep, 13(1), 26-30, 2011.

[6] American Pregnancy Association, "Depression during pregnancy," [Online]. Available:

http://www.americanpregnancy.org/pregnancyhealth/depressiondu ringpregnancy.html. [Accessed June 4, 2012].

[7] The American Congress of Obstetricians and Gynecologists, "Depression during pregnancy: Treatment recommendations. A joint report from APA and ACOG," [Online]. Available: http://www.acog.org/About_ACOG/News_Room/News_Releases/ 2009/Depression_During_Pregnancy. [Accessed June 4, 2012].

[8] Bonari, L., Pinto, N., Ahn, E., Einarson, A., Steiner, M. and Koren, $\mathrm{G}$, "Perinatal risks of untreated depression during pregnancy," Can J Psychiatry, 49(11), 726-735, 2004.

[9] Yonkers, K.A., Wisner, K.L., Stewart, D.E., Oberlander, T.F., Dell, D.L., Stotland, N., Ramin, S., Chaudron, L. and Lockwood, $\mathrm{C}$, "The management of depression during pregnancy: A report from the american psychiatric association and the american college of obstetricians and gynecologists," Gen Hosp Psychiatry, 31(5), 403-413, 2009.

[10] Wisner, K.L., Sit, D.K., Hanusa, B.H., Moses-Kolko, E.L., Bogen, D.L., Hunker, D.F., Perel, J.M., Jones-Ivy, S., Bodnar, L.M. and Singer, L.T, "Major depression and antidepressant treatment: Impact on pregnancy and neonatal outcomes," Am J Psychiatry, 166(5), 557-566, 2009.

[11] Cohen L.S., Altshuler L.L., Harlow B.L., at el. "Relapse of major depression during pregnancy in women who maintain or discontinue antidepressant treatment," JAMA,; 295(5),499-507, 2006.

[12] Dominguez R.A. and Goodnick P.J, "Adverse events after the abrupt discontinuation of paroxetine," Pharmacotherapy, 15(6): 778-780, 1995.

[13] Rosenbaum J.F. and Zajecka J, "Clinical management of antidepressant discontinuation," J Clin Psychiatry, 58 (Suppl 7), 37-40,1997.

[14] Lorant, V., Croux, C., Weich, S., Deliege, D., Mackenbach, J. and Ansseau, M, "Depression and socio-economic risk factors: 7-year longitudinal population study," Br J Psychiatry, 190,293-298, 2007.

[15] Roy-Byrne, P.P., Joesch, J.M., Wang, P.S. and Kessler, R.C, "Low socioeconomic status and mental health care use among respondents with anxiety and depression in the NCS-R," Psychiatr Serv, 60(9), 1190-1197, 2009.
[16] Hayes, R.M., Wu, P., Shelton, R.C., Cooper, W.O., Dupont, W.D, Mitchel, E. and Hartert, T.V, "Maternal antidepressant use and adverse outcomes: A cohort study of 228,876 pregnancies," Am J Obstet Gynecol, 207(1), 49.e1-49.e9, 2012.

[17] Alwan, S., Reefhuis, J., Rasmussen, S.A. and Friedman, J.M, "National Birth Defects Prevention Study. Patterns of antidepressant medication use among pregnant women in a united states population," J Clin Pharmacol, 51(2), 264-270, 2011.

[18] Dietz, P.M., Williams, S.B., Callaghan, W.M., Bachman, D.J., Whitlock, E.P. and Hornbrook, M.C, "Clinically identified maternal depression before, during, and after pregnancies ending in live births," Am J Psychiatry, 164(10):1515-1520, 2007.

[19] Andrade, S.E., Raebel, M.A., Brown, J., Lane, K., Livingston, J., Boudreau, D., Rolnick, S.J., Roblin, D., Smith, D.H., Willy, M.E., Staffa, J.A. and Platt, R, "Use of antidepressant medications during pregnancy: A multisite study," Am J Obstet Gynecol, 198(2), 194.e1-194.e5, 2008.

[20] Cooper, W.O., Willy, M.E., Pont, S.J. and Ray, W.A, "Increasing use of antidepressants in pregnancy," Am J Obstet Gynecol, 196(6), 544.e1-544.e5, 2007.

[21] Chambers, C.D., Hernandez-Diaz, S., Van Marter, L.J., Werler, M.M., Louik, C., Jones, K.L. and Mitchell, A.A, "Selective serotonin-reuptake inhibitors and risk of persistent pulmonary hypertension of the newborn," N Engl J Med, 354(6), 579-587, 2006.

[22] Kallen. B.A. and Otterblad Olausson, P., "Maternal use of selective serotonin re-uptake inhibitors in early pregnancy and infant congenital malformations," Birth Defects Res A Clin Mol Teratol, 79(4), 301-8, 2007.

[23] Kendall-Tackett, K. and Hale, T.W, "The use of antidepressants in pregnant and breastfeeding women: A review of recent studies," $J$ Hum Lact, 26(2), 187-195, 2010.

[24] Pedersen, L.H., Henriksen, T.B., Vestergaard, M., Olsen, J., Bech, B.H, "Selective serotonin reuptake inhibitors in pregnancy and congenital malformations: Population based cohort study," BMJ, 339, b3569, 2009

[25] Toh, S., Mitchell, A.A., Louik, C., Werler, M.M., Chambers, C.D and Hernandez-Diaz, S, "Selective serotonin reuptake inhibitor use and risk of gestational hypertension," Am J Psychiatry, 166(3), 320-328, 2009.

[26] Wen, S.W., Yang, Q., Garner, P., Fraser, W., Olatunbosun, O., Nimrod, C. and Walker, M, "Selective serotonin reuptake inhibitors and adverse pregnancy outcomes," Am J Obstet Gynecol, 194(4), 961-966, 2006.

[27] Ramos, E., Oraichi, D., Rey, E., Blais, L. and Berard, A, "Prevalence and predictors of antidepressant use in a cohort of pregnant women," BJOG, 114(9), 1055-1064, 2007.

[28] Ververs, T., Kaasenbrood, H., Visser, G., Schobben, F., de Jongvan den Berg, L. and Egberts, T, "Prevalence and patterns of antidepressant drug use during pregnancy," Eur J Clin Pharmacol, 62(10):863-870, 2006.

[29] Plant, E.A. and Sachs-Ericsson, N, "Racial and ethnic differences in depression: The roles of social support and meeting basic needs," J Consult Clin Psychol, 72(1), 41-52, 2004.

[30] Hernandez, A., Plant, E.A., Sachs-Ericsson, N., and Joiner, T.E, "Mental health among hispanics and caucasians: Risk and protective factors contributing to prevalence rates of psychiatric disorders," J Anxiety Disord, 19(8), 844-860, 2005.

[31] Sleath, B., West, S., Tudor, G., Perreira, K., King, V. and Morrissey, J, "Ethnicity and prenatal depression: Women's experiences and perspectives on communicating about their emotions and feelings during pregnancy," Patient Educ Couns, 58(1), 35-40, 2005.

[32] Henshaw, E.J., Flynn, H.A., Himle, J.A., O'Mahen, H.A., Forman, J. and Fedock, G, "Patient preferences for clinician interactional style in treatment of perinatal depression," Qual Health Res, 21(7), 936-951, 2011.

[33] O'Mahen, H.A. and Flynn, H.A, "Preferences and perceived barriers to treatment for depression during the perinatal period," $J$ Womens Health (Larchmt), 17(8), 1301-1309, 2008.

[34] Melfi, C.A., Chawla, A.J., Croghan, T.W., Hanna, M.P., Kennedy, S., and Sredl, K, "The effects of adherence to antidepressant treatment guidelines on relapse and recurrence of depression," Arch Gen Psychiatry, 55(12):1128-1132, 1998. 